\title{
GALAZZI Enrica. 2002. Le son à l'école. Phonétique et enseignement des langues (fin XIXe siècle - début XXe siècle). Milano : Editrice La Scuola.
}

\section{Elisabet Hammar}

\section{(2) OpenEdition Journals}

Édition électronique

URL : https://journals.openedition.org/dhfles/2331

DOI : $10.4000 /$ dhfles.2331

ISSN : 2221-4038

Éditeur

Société Internationale pour l'Histoire du Français Langue Étrangère ou Seconde

Édition imprimée

Date de publication : 1 décembre 2002

Pagination : 177-180

ISSN : 0992-7654

Référence électronique

Elisabet Hammar, « GALAZZI Enrica. 2002. Le son à l'école. Phonétique et enseignement des langues (fin XIXe siècle - début XXe siècle). Milano : Editrice La Scuola. », Documents pour I'histoire du français langue étrangère ou seconde [En ligne], 29 | 2002, mis en ligne le 15 mai 2014, consulté le 27 mai 2021. URL : http://journals.openedition.org/dhfles/2331 ; DOI : https://doi.org/10.4000/dhfles.2331

Ce document a été généré automatiquement le 27 mai 2021.

(c) SIHFLES 


\title{
GALAZZI Enrica. 2002. Le son à l'école. Phonétique et enseignement des langues (fin XIXe siècle - début XXe siècle). Milano : Editrice La Scuola.
}

\author{
Elisabet Hammar
}

1 Depuis une bonne dizaine d'années, les travaux d'Enrica Galazzi font autorité, au sein de la SIHFLES, en ce qui concerne l'histoire de la phonétique et de l'enseignement de la prononciation du français. L'ouvrage nous offre des textes jusque-là dispersés dans des publications différentes, non seulement réunis, mais remodelés et complétés.

2 La phonétique moderne est une science qui s'est développée dans une large mesure à l'intérieur des frontières françaises, par le biais d'un Paul Passy ou d'un abbé Rousselot et Enrica Galazzi concentre ses recherches sur le côté francophone. Elle parle moins d'un Sweet, d'un Jones, d'un Viëtor ou d'un Jespersen. Même si le français a longtemps été la langue enseignée de préférence dans le monde occidental et même si ce qui vaut pour le français vaut souvent pour d'autres langues, il est question d'une période où les pays européens ont commencé à suivre des chemins divergents en ce qui concerne l'organisation scolaire et l'enseignement des langues modernes. Il aurait donc été de mise, dans le texte, de souligner, de temps à autre, qu'il s'agit surtout de la langue française et de la vision française de cette période passionnante dans l'histoire de l'enseignement des langues.

3 Centrée sur l'oral, la phonétique visait le monde pratique mais comme elle s'appuyait toujours sur des observations précises, cela la rendait respectable dans le monde académique. La parole étant devenue un objet de recherches, les professeurs pouvaient s'y intéresser sans être assimilés à des "vauriens » acédémiques (enseigner à parler une langue a longtemps été considéré comme une occupation peu sérieuse). Dans le monde scolaire, la science de la phonétique avait ouvert une brèche dans la défense coriace du 
vieil humanisme européen, celui qui ne jurait que par le patrimoine commun, l'héritage de l'Antiquité; elle avait ouvert la voie au plurilinguisme, à la conscience des différences entre les cultures européennes et au sens de la primauté de la communication orale. La victoire ne serait cependant ni rapide, ni aisée.

Enrica Galazzi commence par évoquer les débuts de la phonétique, quand les savants ne visaient pas encore la pédagogie, mais s'intéressaient surtout au côté diachronique, c'est-à-dire aux lois phonétiques réglant l'évolution des langues, ou bien à la dialectologie. A peine vingt ans après l'invention du terme "phonétique» pour désigner la science des sons parlés, son application pratique était déjà discutée à l'intérieur du mouvement européen dit "de la réforme », notamment la réforme de l'enseignement des langues vivantes. L'époque était mûre : quatre ans après le cri de guerre de Viëtor, en 1882, Der Sprachunterricht muss umkehren! [l'enseignement des langues doit faire volte-face !] qui est souvent considéré comme le point de départ de ce mouvement, trois événements avaient consolidé les nouvelles idées et montré au monde ce qui était en cours : la création par Paul Passy de la revue Le Maître Phonétique et de l'association "mère", l'Association phonétique internationale, le Congrès philologique de Stockholm, qui à son tour engendra une association scandinave en faveur d'une réforme, le "Quousque Tandem»; tous trois pendant l'année 1886. Le premier point préconisé par les adeptes de la réforme était d'enseigner la prononciation à l'aide de la phonétique et de la transcription phonétique issue de cette science.

5 Paul Passy, l'un des grands initiateurs, était présent à ce congrès et, de retour en France, en rendit compte au ministre de l'Instruction publique. Enrica Galazzi trace amoureusement le portrait de cet idéaliste socialiste et chrétien, ainsi que celui du fondateur de la phonétique expérimentale, l'abbé Rousselot. Les conférences de ces deux personnages extraordinaires, en France, à Greifswald, à Marburg, à Genève, etc., le plus souvent sous forme de cours d'été pour des professeurs venant de presque tous les pays européens, ont propagé les expériences des pionniers et enseigné les pratiques de l'application des découvertes, à l'aide d'instruments ou non. D'autres enthousiastes, connus ou moins connus, sont passés en revue: Théodore Rosset, à l'université de Grenoble, Adolphe Zünd-Burguet, Kristoffer Nyrop (Ton thé t'a-t-il ôté ta toux ?), Felix Franke et beaucoup d'autres. L'auteur parle dans un chapitre des machines qui apprennent à parler et des machines qui parlent: le palais artificiel, le tambour inscripteur, le pneumographe, le signal du larynx, les guide-langue, etc. et, bien sûr, le phonographe. Un autre chapitre est consacré à l'élaboration qui se fit non sans difficulté de l'Alphabet Phonétique International : fallait-il tenir compte de toutes les variantes, à l'intérieur d'une même langue ou entre les différentes langues (tâche matériellement impossible), ou fallait-il viser un but pratique, noter grossièrement chaque son et compléter ensuite cette notation avec des descriptions plus détaillées pour chaque langue et selon la position de chaque son?

Dans ce milieu favorable à la langue parlée, il eut été étonnant que l'idée d'une grammaire de la langue parlée ne vint pas à l'esprit. Paul Passy, après avoir été, dans Le Maître Phonétique, assez sévère à l'égard de quelques essais antérieurs, publia en 1893, avec Frantz Beyer, Elementarbuch des Gesprochene Französisch, mais malheureusement Enrica Galazzi ne nous fait pas part du contenu de cet ouvrage, qui reçut pourtant, semble-t-il, un bon accueil. Elle nous donne cependant deux exemples de "grammaires phonétiques" afin de permettre d'évaluer "les ambitions et les limites de cette 
entreprise». Il s'agit du Précis d'orthographe et de grammaire phonétique pour l'enseignement $d u$ français à l'étranger de Léon Clédat et de l'« Essai de grammaire phonétique à l'intention des élèves étrangers désireux de parler, écrire, lire le français » de Gustav Rolin, l'un paru en 1890, l'autre, opuscule d'une quarantaine de pages, publié dans deux numéros consécutifs de Phonetische Studien, en 1891 et 1892. Les deux ouvrages, conçus par des personnages originaux, furent jugés par Passy, le premier comme " tout à fait malheureux ", le second comme la première ébauche d'une grammaire du français parlé. Clédat voulait surtout réformer l'orthographe par les acquis de la phonétique, mais n'allait pas beaucoup plus loin, d'après les critiques, tandis que Rolin, après avoir présenté une analyse assez poussée de la syllabation orale, avait bousculé l'ordre grammatical traditionnel, parfois pour des raisons nécessaires à une grammaire de la langue parlée, parfois pour des raisons moralisatrices, jusqu'à placer les pronoms possessifs le plus tard possible pour contrer leur charge " égoïste». Pour finir, Enrica Galazzi reprend la discussion de l'époque sur les meilleurs modèles de prononciation à proposer aux étrangers, choix qui s'imposent dès que la langue parlée vient en premier lieu.

7 Enrica Galazzi termine son remarquable ouvrage par une étude de cas, concernant la réception italienne de toutes ces idées nouvelles pour l'enseignement des langues vivantes dans les écoles. Ceci donne du relief à l'idéalisme, l'enthousiasme et l'esprit de découverte exposés aux pages précédentes. Comment aboutit toute cette énergie d'argumentation et de persuasion? Comment arriver à vaincre la résistance conservatrice, les lois de la paresse? Enrica Galazzi nous le montre, avec la même honnêteté scientifique que celle dont elle fait preuve tout au long de son livre et avec cette volonté tenace - et heureuse - de capter toutes les nuances et d'arriver au plus près de la compréhension et de la vérité d'une période fascinante et charnière dans l'histoire de l'enseignement des langues.

\section{AUTEUR}

\section{ELISABET HAMMAR}

Université de Linköping (Suède) 